\title{
Improving bearings-only target state estimation tracking problem by using adaptive and nonlinear kalman algorithms
}

\author{
Tammam Khadour, Michel Al Saba, Louay Saleh
}

Department of Electro-Mechanical Engineering, Higher Institute for Applied Sciences and Technology, Syria

\begin{tabular}{l}
\hline \hline Article Info \\
\hline Article history: \\
Received Dec 02, 2018 \\
Revised Jan 21, 2019 \\
Accepted Mar 10, 2019 \\
\hline Keywords: \\
AKF \\
ANKF \\
CKF \\
EKF \\
Estimation \\
Noise \\
Nonlinear \\
Sensor \\
Target tracking \\
UKF \\
\hline
\end{tabular}

\begin{abstract}
Finding the best estimate of the process state from noisy data is the main problem in tracking systems, many efforts and researches have been done to remove this noise. More useful information about the target's state can be extracted from observations by using a more appropriate model for the target's motion or using additional sensors. In this paper, we will introduce two methods to improve the estimation of bearing-only target tracking problem in two dimensions (2D). The first method is by adding a third sensor and making a good alignment of those sensors, and at the same time an extended Kalman filter (EKF), unscented Kalman filter (UKF) and cubature Kalman filter (CKF) are implemented. The second method is by applying an adaptive nonlinear Kalman filter (ANKF) for two sensors to solve the problem of measurement variance uncertainty.

Copyright $\odot 2019$ Institute of Advanced Engineering and Science. All rights reserved.
\end{abstract}

Corresponding Author:

Tammam Khadour,

Department of Electro-Mechanical Engineering,

Higher Institute for Applied Sciences and Technology,

Damascus, Syria- BP 31983.

Email: tammam.khaddour@hiast.edu.sy

\section{INTRODUCTION}

Different types of vehicles like cars, ships, planes perform different types of maneuvers from simple to complex. A single model does not represent all such maneuvers and particular multiple models are constructed to accurately describe and aid in predicting the trajectory according to the application [1].

Many specific dynamic models of target motion have been developed for maneuvering target tracking. The simplest model for a maneuvering target are the white-noise acceleration model [2]. Many target tracking algorithms are proposed based on so many different filters, such as alpha-beta filter, Gaussian sum filter. nonlinear Kalman filter and adaptive Kalman filter as in [3-4]. The Kalman filter is used for state estimation of some linear dynamic system [5-6], which can estimate the future state of the signal based on the signal statistical characteristic, the algorithm is formulated in two steps: prediction and updating. More common methods for dealing with a nonlinear model is to use the extended Kalman filter (EKF) [7].

Results of implementing non-linear Kalman filter for 2D tracking problems using two sensors showed that there are problems when the target is near the straight line which is between the two sensors [8]. The UKF algorithm is based on the Unscented Transform (UT) for computing the mean and covariance matrix of a variable, it is far easier to implement than the EKF [9]. For system model with a good linear and quadratic approximations the EKF works well but if the system is more nonlinear the UKF is often superior over EKF, the CKF algorithm is used in many applications like simultaneous localization and mapping [10], and in improving the tracking performance [11]. A Gaussian-sum cubature Kalman filter with improved 
robustness demonstrated good accuracy and efficiency for the bearings-only tracking problem compared to the original algorithm of CKF [12]. In adaptive Kalman filter (AKF) the filter adjusts its knowledge about the mean and covariance matrix values according to the gap between the predicted estimations and the current measurements. A new methodology is proposed based on the use of weighted least squares methodology instead of traditional least square approach [13]. The estimation of parameter and state of nonlinear highspeed objects by adaptive unscented Kalman filter is proposed in [14]. The navigation system depends on sensors output, where the navigation output must be accurate and robust with erroneous measurements which caused from noise, that makes the noise process very important for sensors and control process in mobile robot [15]. Using a mobile robot for following sound sources in real time depending on an active virtual impedance algorithm for obstacle avoidance where the new algorithm changes the impedance dynamically in two cases: one distance sensor and two distance sensors, using more ultrasonic sensors for future research [16]. Implemented binary motion sensors for the problem of automated position estimation, using Passive Infrared (PIR) motion sensors with the suggested estimator, develop the detection system by different sensor models and arrangements is a future work [17]. A very important Kalman Filter use is to reduce the noise on the accelerometer and gyroscope sensors for quadrotor navigation system, where the bias and zero velocity compensations are able to improve the position estimation of the quadrotor by reducing the effects of drift due to integration [18].

The contributions of this study are the development in bearings-only target state estimation tracking problem by using many Kalman Filter algorithms (EKF, UKF, CKF) to reduce the noise on the sensor, improving the accuracy of estimation by adding a third sensor as hardware solution, which avoid the target movement on the straight line between two sensors, and change the alignment of those three sensors to have an accurate estimation. Another solution is software depending on [19] which is based on the innovationbased adaptive estimation (IAE) approach and the adaptive fading Kalman filter (AFKF) approach. This method was used to solve measurement variance uncertainty with two sensors in linear states. In this paper we will use the algorithms presented in [19] but we will change the flow chart of the (IAE, AFKF) algorithms to be suitable for nonlinear measurement as adaptive nonlinear Kalman filter (ANKF) for two sensors. Results will then be compared.

The structure of this paper is as follows: Section two presents the bearing-only tracking model. Section three describes nonlinear Kalman filters (EKF, UKF, and CKF). Section four concerns proposed method using Adaptive Kalman filter implementation. In section five simulation results are compared (EKF, UKF, and CKF) in two sensors case, three sensors case and using proposed ANKF with two sensors case. Finaly conclusion will be presented in section six.

\section{BEARING-ONLY TRACKING MODEL}

Target motion and sensor measurement models in the two dimensional (2D) case are considered. In cartesian coordinates, the target state $\mathrm{e}_{\mathrm{k}}$ with position $\left(x_{k}, y_{k}\right)$ and velocity $\left(\dot{x}_{k}, \dot{y}_{k}\right)$ at time $\mathrm{k}$ is expressed as [8]:

$$
e_{k}=\left(\begin{array}{llll}
x_{k} & y_{k} & \dot{x}_{k} & \dot{y}_{k}
\end{array}\right)^{T}
$$

The dynamics of the target is modeled as a linear, discretized Wiener velocity model:

$$
e_{k}=\left(\begin{array}{cccc}
1 & 0 & T & 0 \\
0 & 1 & 0 & T \\
0 & 0 & 1 & 0 \\
0 & 0 & 0 & 1
\end{array}\right) e_{k-1}+v_{k-1}
$$

Where $v_{k-1}$ is Gaussian process noise with zero mean and covariance:

$$
Q_{k-1}=\left(\begin{array}{cccc}
\frac{1}{3} T^{3} & 0 & \frac{1}{2} T^{2} & 0 \\
0 & \frac{1}{3} T^{3} & 0 & \frac{1}{2} T^{2} \\
\frac{1}{2} T^{2} & 0 & T & 0 \\
0 & \frac{1}{2} T^{2} & 0 & T
\end{array}\right) \sigma_{v}^{2}
$$

\footnotetext{
Improving bearings-only target state estimation tracking problem by using adaptive... (Tammam Khadour)
} 
Where $\mathrm{T}$ is the sampling time and $\sigma_{v}^{2}$ is the spectral density of the noise. The measurement model for sensor $i$ is defined as:

$$
z_{k}=h\left(e_{k}, k\right)=\left(\begin{array}{c}
\theta_{k}^{1} \\
\theta_{k}^{2}
\end{array}\right)+w_{k}
$$

Where $\theta_{k}^{i}=\arctan \left(\frac{y_{k}-s_{y}^{i}}{x_{k}-s_{x}^{i}}\right)+w_{k}^{i}$ and $\left(s_{x}^{i}, s_{y}^{i}\right)$ is the position of sensor $\mathrm{i}$ and $w_{k}^{i} \sim N\left(0, \sigma_{w}^{2}\right)$.

\section{NONLINEAR KALMAN FILTER}

\subsection{Extended Kalman Filter (EKF)}

The extended Kalman is the nonlinear version of the Kalman filter which linearizes about an estimate of the current mean and covariance. First order extended Kalman filters are presented. The EKF model is [4], [8]:

$$
\left\{\begin{array}{l}
e_{k}=f\left(e_{k-1}, k-1\right)+v_{k-1} \\
z_{k}=h\left(e_{k}, k\right)+w_{k}
\end{array}\right.
$$

Where the processing and measurement noise are white Gaussian with:

$$
\left\{\begin{array}{l}
v_{k-1} \sim N\left(0, Q_{k-1}\right) \\
w_{k} \sim N\left(0, R_{k}\right)
\end{array}\right.
$$

The EKF algorithm:

a. Prediction

$$
\left\{\begin{array}{c}
m_{k}^{-}=f\left(m_{k-1}, k-1\right) \\
P_{k}^{-}=F_{e}\left(m_{k-1}, k-1\right) P_{k-1} F_{e}^{T}+Q_{k-1} \\
{\left[F_{e}(m, k-1)\right]_{j, i}=\left.\frac{\partial f_{j}(e, k-1)}{\partial x_{i}}\right|_{e=m}}
\end{array}\right.
$$

b. Update

$$
\begin{aligned}
& \left\{\begin{array}{c}
\frac{\partial \mathrm{h}^{\mathrm{i}}\left(\mathrm{e}_{\mathrm{k}}\right)}{\partial \mathrm{x}_{\mathrm{k}}}=\frac{-\left(\mathrm{y}_{\mathrm{k}}-\mathrm{s}_{\mathrm{y}}^{\mathrm{i}}\right)}{\left(\mathrm{x}_{\mathrm{k}}-\mathrm{s}_{\mathrm{x}}^{\mathrm{i}}\right)^{2}+\left(\mathrm{y}_{\mathrm{k}}-\mathrm{s}_{\mathrm{y}}^{\mathrm{i}}\right)^{2}} \\
\frac{\partial \mathrm{h}^{\mathrm{i}}\left(\mathrm{e}_{\mathrm{k}}\right)}{\partial \mathrm{y}_{\mathrm{k}}}=\frac{-\left(\mathrm{x}_{\mathrm{k}}-\mathrm{s}_{\mathrm{x}}^{\mathrm{i}}\right)}{\left(\mathrm{x}_{\mathrm{k}}-\mathrm{s}_{\mathrm{x}}^{\mathrm{i}}\right)^{2}+\left(\mathrm{y}_{\mathrm{k}}-\mathrm{s}_{\mathrm{y}}^{\mathrm{i}}\right)^{2}} \\
\frac{\partial \mathrm{h}^{\mathrm{i}}\left(\mathrm{e}_{\mathrm{k}}\right)}{\partial \dot{\mathrm{x}}_{\mathrm{k}}}=0 .
\end{array}\right. \\
& \left\{\begin{array}{c}
u_{k}=z_{k}-h\left(m_{k}^{-}, k\right) \\
S_{k}=H_{e}\left(m_{k}^{-}, k\right) P_{k}^{-} H_{e}^{T}\left(m_{k}^{-}, k\right)+R_{k} \\
K_{k}=P_{k}^{-} H_{e}^{T}\left(m_{k}^{-}, k\right) S_{k}^{-1} \\
m_{k}=m_{k}^{-}+K_{k} u_{k} \\
P_{k}=P_{k}^{-}-K_{k} S_{k} K_{k}^{T} \\
{\left[H_{e}(m, k)\right]_{j, i}=\left.\frac{\partial h_{j}(e, k)}{\partial x_{i}}\right|_{e=m}}
\end{array}\right.
\end{aligned}
$$

The $m_{k}^{-}$and $P_{k}^{-}$are the predicted mean and covariance of the state. 


\subsection{Unscented Kalman Filter (UKF)}

In models with high non-linear predict and update functions $(f, h) \mathrm{EKF}$ gives particularly poor performance because the covariance is propagated through linearization of the underlying non-linear model. The unscented Kalman filter (UKF) is a sampling method uses a deterministic sampling technique known as the unscented transform (UT) to pick a minimal set of $(2 n+1)$ sample points called (sigma points) around the mean of the n-dimensional distribution of the covariance matrix, where computational efficiency is the advantage of the (UT). The UKF algorithm [4], [8], [9]:

a) Sigma point

The $n$-dimensional $e_{k}$ with mean $\hat{e}_{k / k}$ and covariance $P_{k / k}$ is approximated by $2 n+1$ weighted samples named sigma points selected using the following algorithm:

$$
\left\{\begin{array}{l}
\chi_{k / k}^{0}=\hat{e}_{k / k}, \quad W_{0}=\kappa /(n+\kappa) \\
\chi_{k / k}^{i}=\hat{e}_{k / k}+\left(\sqrt{(n+\kappa) P_{k / k}}\right)_{i}, \quad W_{i}=1 / 2(n+\kappa) \\
\chi_{k / k}^{i+n}=\hat{e}_{k / k}-\left(\sqrt{(n+\kappa) P_{k / k}}\right)_{i}, \quad W_{i+n}=1 / 2(n+\kappa)
\end{array}\right.
$$

where $\kappa$ is a tuning parameter of the filter which is recommended to be chosen such that $\kappa+n=3$, $\left(\sqrt{(n+\kappa) P_{k / k}}\right)_{i}, i=1, \cdots, n$, is the $i^{\text {th }}$ row or column of the matrix square root of $(n+\kappa) P_{k / k}$, and $W_{i}$ is the weight associated with the $i^{\text {th }}$ point.

b) Prediction

$$
\left\{\begin{array}{c}
\chi_{k+1 / k}^{i}=f\left(\chi_{k / k}^{i}, k\right) \\
\hat{e}_{k+1 / k}=\sum_{i=0}^{2 n} W_{i} \chi_{k+1 / k}^{i} \\
P_{k+1 / k}=Q_{k}+\sum_{i=0}^{2 n} W_{i}\left(\chi_{k+1 / k}^{i}-\hat{e}_{k+1 / k}\right)\left(\chi_{k+1 / k}^{i}-\hat{e}_{k+1 / k}\right)^{T}
\end{array}\right.
$$

c) Update

$$
\begin{gathered}
\left\{\begin{array}{c}
Z_{k+1}^{i}=h\left(\chi_{k+1}^{i}\right) \\
\hat{z}_{k+1}=\sum_{i=0}^{2 n} W_{i} Z_{k+1}^{i} \\
\hat{e}_{k+1 / k+1}=\hat{e}_{k+1 / k}+K_{k+1}\left(z_{k+1}-\hat{z}_{k+1}\right) \\
P_{k+1 / k+1}=P_{k+1 / k}-K_{k+1} P_{k+1}^{v v} K_{k+1}^{T}
\end{array}\right. \\
\left\{\begin{array}{c}
P_{k+1}^{v v}=\sum_{i=0}^{2 n} W_{i}\left(Z_{k+1}^{i}-\hat{z}_{k+1}\right)\left(Z_{k+1}^{i}-\hat{z}_{k+1}\right)^{T}+R_{k+1} \\
P_{k+1}^{x v}=\sum_{i=0}^{2 n} W_{i}\left(\chi_{k+1 / k}^{i}-\hat{e}_{k+1}\right)\left(\chi_{k+1 / k}^{i}-\hat{e}_{k+1}\right)^{T} \\
K_{k+1}=P_{k+1}^{x v}\left(P_{k+1}^{v v}\right)^{-1}
\end{array}\right.
\end{gathered}
$$

\subsection{Cubature Kalman Filter (CKF)}

The CKF is a Kalman-filter-based algorithm that uses the third-degree spherical-radial rule to generate cubature points with normalized weights to numerically approximate the multidimensional integrals involved in Bayesian filtering [8], [10], [11], [12]:

a) Prediction

- Calculate the cubature points from the intersection of $n$-dimension unit sphere and the Cartesian axes $\mathrm{c}_{\mathrm{i}}$ :

$$
\xi_{i}=\left\{\begin{array}{rl}
\sqrt{n} c_{i} & i=1, \ldots, n \\
-\sqrt{n} c_{i-n} & i=n+1, \ldots, 2 n
\end{array}\right.
$$


- Propagate the cubature points:

$$
X_{i, k-1 / k-1}=\sqrt{P_{k-1 / k-1}} \xi_{i}+m_{k-1 / k-1}
$$

- Evaluate the cubature points with the dynamic model function:

$$
X_{i, k-1 / k-1}^{*}=f\left(X_{i, k-1 / k-1}\right)
$$

- Estimate the predicted state mean:

$$
m_{k / k-1}=\frac{1}{2 n} \sum_{i=1}^{p^{n}} X_{i, k-1 / k-1}^{*}
$$

- Estimate the predicted error covariance:

$$
\begin{aligned}
P_{k / k-1} & =\frac{1}{2 n} \sum_{i=1}^{p^{n}} X_{i, k-1 / k-1}^{*} X_{i, k-1 / k-1}^{{ }^{*} T} \\
& -m_{k / k-1} m_{k / k-1}^{T}+Q_{k-1}
\end{aligned}
$$

b) Update

- Calculate the cubature points from the intersection of n-dimension unit sphere and the Cartesian.

- Propagate the cubature points:

$$
X_{i, k / k-1}=\sqrt{P_{k / k-1}} \xi_{i}+m_{k / k-1}
$$

- Evaluate the cubature points with the measurement model function:

$$
Z_{i, k / k-1}^{*}=f\left(X_{i, k / k-1}\right)
$$

- Estimate the predicted measurement:

$$
\hat{z}_{k / k-1}=\frac{1}{2 n} \sum_{i=1}^{p^{n}} Z_{i, k / k-1}^{*}
$$

- Estimate the innovation covariance matrix:

$$
\begin{aligned}
S_{k / k-1} & =\frac{1}{2 n} \sum_{i=1}^{p^{n}} Z_{i, k / k-1}^{*} Z_{i, k / k-1}^{* T} \\
& -\hat{z}_{k / k-1} \hat{z}_{k / k-1}^{T}+R_{k}
\end{aligned}
$$

- Estimate the cross-covariance matrix:

$$
\begin{aligned}
P_{x y, k / k-1} & =\frac{1}{2 n} \sum_{i=1}^{p^{n}} X_{i, k-1 / k-1}^{*} Z_{i, k / k-1}^{*_{T}} \\
- & m_{k / k-1} \hat{z}_{k / k-1}^{T}
\end{aligned}
$$

- Calculate the Kalman gain term and the smoothed state mean and covariance:

$$
\left\{\begin{array}{c}
K_{k}=P_{x y, k / k-1} S_{k / k-1}^{-1} \\
m_{k / k}=m_{k / k-1}+K_{k}\left(z_{k}-\hat{z_{k / k-1}}\right) \\
P_{k / k}=P_{k / k-1}-K_{k} P_{y y, k / k-1} K_{k}^{T}
\end{array}\right.
$$




\section{PROPOSED ALGORITHM - (ANKF)}

One of the KF problems occurs when the noise statistics $\mathrm{Q}$ and $\mathrm{R}$ are unknown, to solve this problem the adaptive Kalman filter is used. In this article we will use two algorithms which are presented in [19] while changing some steps according to nonlinear problem. The innovation-based adaptive estimation (IAE) approach and the adaptive fading Kalman filter (AFKF) approach for Linear Kalman filter are used in [19], where Figures 1 and 2, show those two algorithms. We changed the Block A by Block B and C which are the EKF algorithm for nonlinear problem.

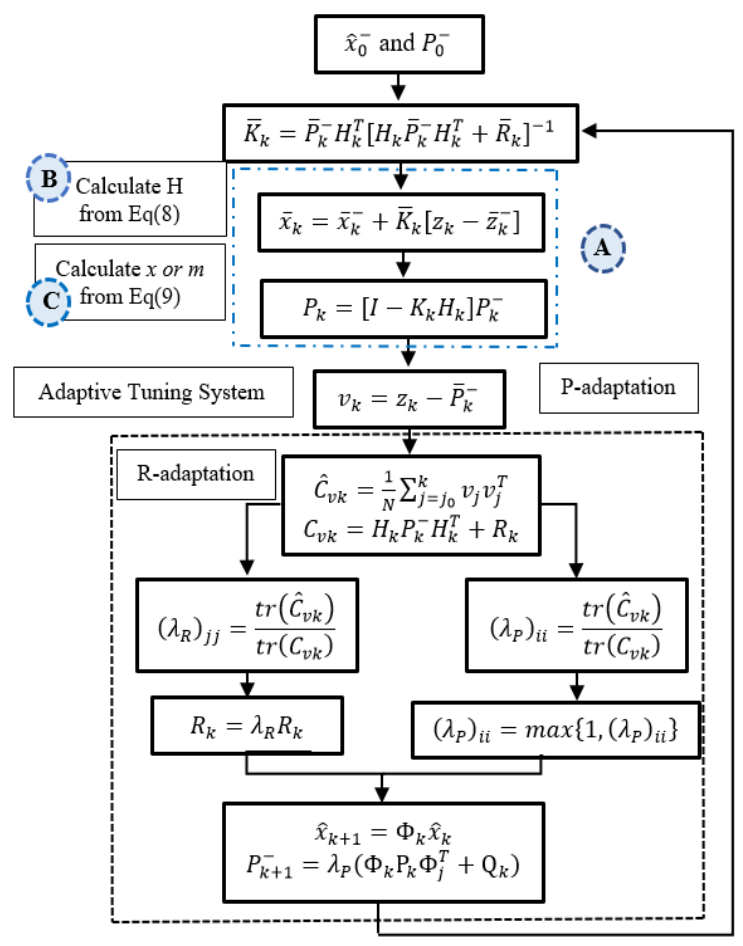

Figure 1. Flow chart of the proposed ANKF1 method

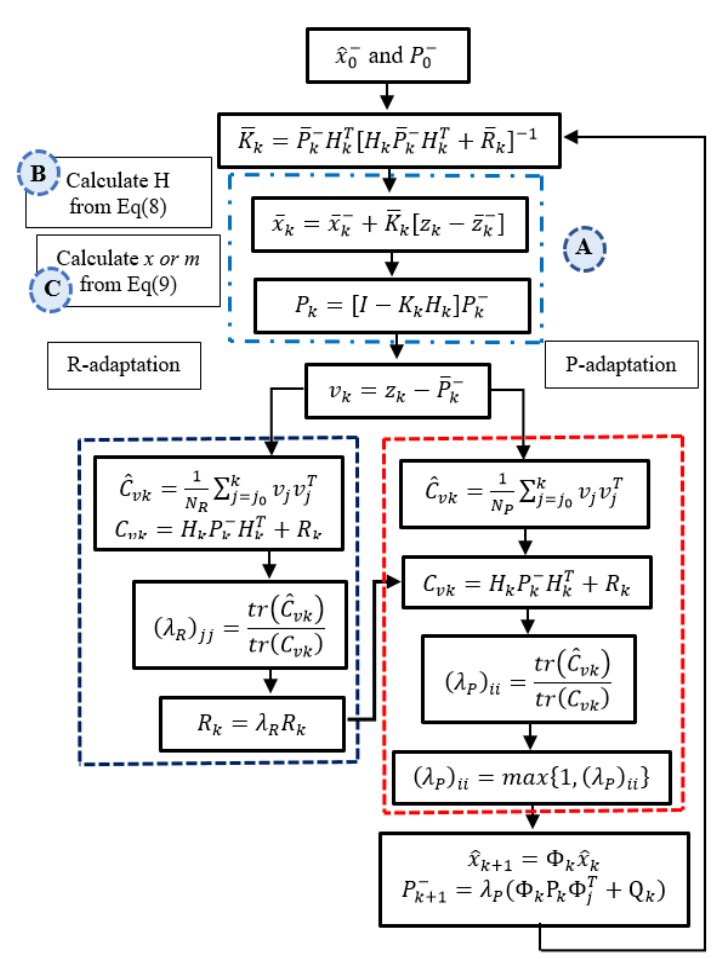

Figure 2. Flow chart of the proposeANKF2 method

\section{Where:}

a. $\lambda_{P}=\operatorname{diag}\left[\lambda_{1} \cdot \lambda_{2} \ldots . \lambda_{m}\right]$ is a factor to option the new $\bar{P}_{k}^{-}$

b. The algorithms of various adaptive fading are depending on $\lambda_{i}$ values:

$$
\left\{\begin{array}{l}
\lambda_{i} \leq 1 \text { steady state processing } \\
\lambda_{i}>1 \text { unstable filtring } \\
\lambda_{i}=1 \text { change to } K F
\end{array}\right.
$$

c. $\bar{C}_{v k}$ : is calculated for deviation due to the changes of covariance and measurement noise.

d. The optimum fading factors are: $\lambda_{i}=\left(\lambda_{P}\right)_{i i}=\max \left\{1 \cdot \frac{\operatorname{tr}\left(\hat{C}_{v k}\right)}{\operatorname{tr}\left(C_{v k}\right)}\right\} ; i=1.2 \ldots . m$

The covariance matrices can be defined as:

$$
\begin{aligned}
& \text { Single factor: } \lambda_{j}=\left(\lambda_{R}\right)_{j j}=\frac{\operatorname{tr}\left(\hat{C}_{v k}\right)}{\operatorname{tr}\left(C_{v k}\right)} ; j=1.2 \ldots . n \\
& \text { Multiple factors: } \lambda_{j}=\left(\lambda_{R}\right)_{j j}=\frac{\operatorname{tr}\left(\hat{C}_{v k}\right)_{j j}}{\operatorname{tr}\left(C_{v k}\right)_{j j}} ; j=1.2 \ldots . n
\end{aligned}
$$

The adaptation measurement is: $\bar{R}_{k}=\lambda_{R} R_{k}$ 


\section{SIMULATION RESULTS}

The EKF, UKF and CKF were applied to estimate the position of a target following two scenarios:

- Scenario1: two sensors.

- Scenario2: three sensors.

The proposed method ANKF was applied for Scenario3 with two sensors. Table 1 shows the values of parameters for the three scenarios.

Table 1. Value of Simulations Parameters

\begin{tabular}{cc}
\hline Parameters & Value \\
\hline$\sigma_{v}^{2}$ & 0.1 \\
$\dot{\sigma}_{v}^{2}$ (uncertainly) for adaptive & 0.01 \\
$\sigma_{w}$ & 0.05 \\
Target start state & {$\left[\begin{array}{lll}0 & 0 & 0\end{array}\right]$} \\
Covariance of the state on the initial time & $\mathrm{P}_{0}=\operatorname{diag}\left[\begin{array}{ll}0.1, & 0.1,10,10\end{array}\right]$ \\
$\mathrm{T}$ & $0.01 \mathrm{~s}$ \\
Monte Carlo runs number & 100 \\
\hline
\end{tabular}

\subsection{Scenario 1:}

$\mathrm{S} 1($ position $)=[1-2] ; \mathrm{S} 2($ position $)=[1-2]$. Figure 3 shows the trajectory and the estimated trajectories. Figure 4 shows a zooming to the Figure 3 because results are so close. Table 2 . shows the comparison between methods depending on Root Mean Square (RMS) error.

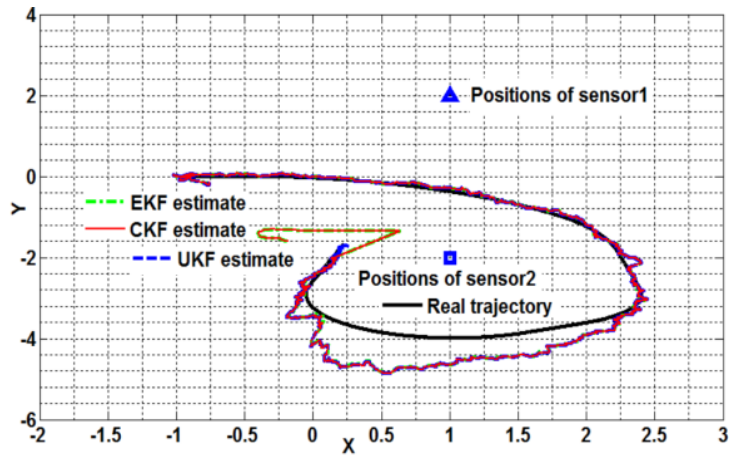

Figure 3. The trajectory of scenario 1

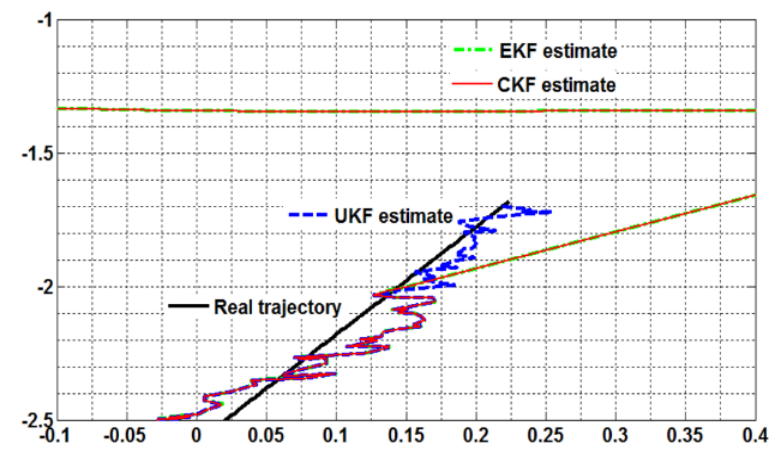

Figure 4. Zooming at the trajectory

Table 2. Comparison between the Methods (Scenario1)

\begin{tabular}{cc}
\hline Method & RMS error \\
\hline EKF & 0.2547 \\
UKF & 0.2243 \\
CKF & 0.2579 \\
\hline
\end{tabular}

\subsection{Scenario 2:}

$\mathrm{S} 1($ position $)=[1-2] ; \mathrm{S} 2($ position $)=[1-2] ; \mathrm{S} 3($ position$)=[1-(2 / \tan (30)), 0]($ we select the position of sensor3 to make equilateral triangle).

Figure 5 shows the real trajectory and the estimated trajectories. Figure 6 shows zooming at Figure 5 because the results are so close Table 3 shows the comparison between methods depending on RMS error.

Table 3. Comparison between the Methods (Scenario2):

\begin{tabular}{cc}
\hline Method & RMS error \\
\hline EKF & 0.13748 \\
UKF & 0.06294 \\
CKF & 0.13768 \\
\hline
\end{tabular}




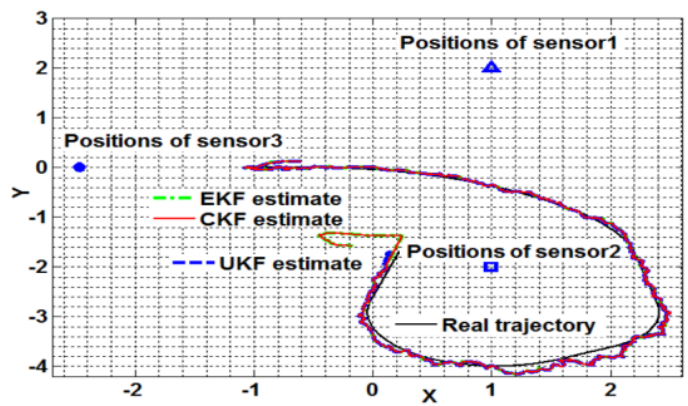

Figure 5. The trajectory of scenario 2

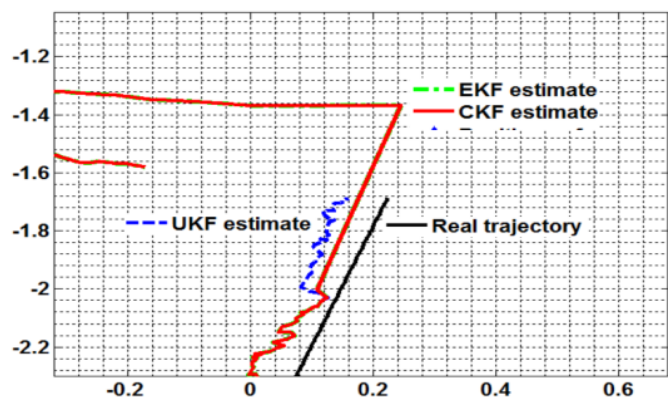

Figure 6. Zooming at the trajectory

As expected, adding a third sensor decreased RMS for all algorithms and the UKF algorithm provides the best results.

\subsection{Scenario 3:}

In this scenario we used the adaptive Kalman filter with nonlinear measurement matrix from [4]:

$$
h\left(x_{k}, k\right)=\left(\begin{array}{l}
\theta_{y} \\
\psi_{z}
\end{array}\right)=\left[\begin{array}{c}
\tan ^{-1}\left(y_{k} / x_{k}\right) \\
\tan ^{-1}\left(z_{k} /{\left.\sqrt{x_{k}^{2}+y_{k}^{2}}\right)}^{2}\right.
\end{array}\right]
$$

The two proposed adaptive Kalman filters algorithms are used, and compared with the most used algorithm EKF. Results are shown on Figures 7 and 8:

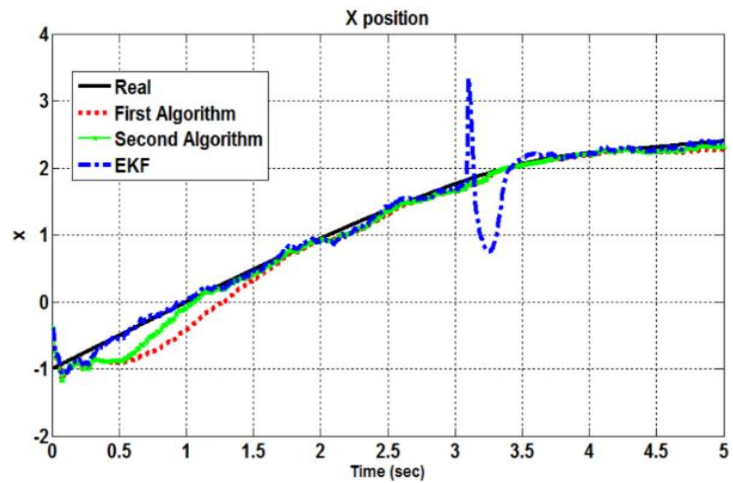

Figure 7. The trajectory of scenario 3

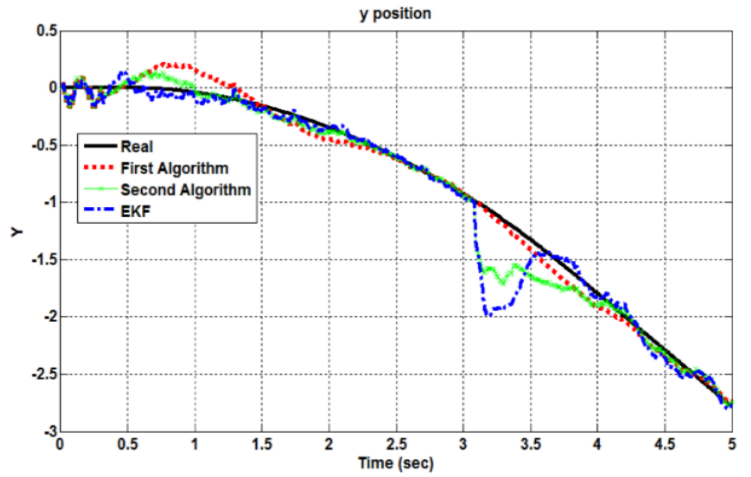

Figure 8. Zooming at the trajectory

We can see from Table 4 that the RMS error decreased by using adaptive Kalman filter with nonlinear measurement matrix comparing with Extended Kalman Filter (EKF). The ANKF2 algorithm provides the best result in estimation better than EKF and ANKF1 with ratio of about $50 \%$.

Table 4. Comparison between the Methods (ANKF, EKF):

\begin{tabular}{cc}
\hline Method & RMS error \\
\hline EKF & 0.5652 \\
ANKF1 & 0.5112 \\
ANKF2 & 0.2730 \\
\hline
\end{tabular}

\section{CONCLUSION}

In this paper, we proposed nonlinear Kalman filter algorithms (EKF, UKF, and CKF) to improve and find the best estimate of the process state from noisy data, in bearings-only target state estimation tracking problem in three scenarios. In the first scenario we applied the past three algorithms with two sensors case, where the UKF gave the best RMS error by Monte Carlo 100 runs number. In the second

Improving bearings-only target state estimation tracking problem by using adaptive... (Tammam Khadour) 
scenario we applied the algorithms and added a third sensor making equilateral triangle, the results showed that the RMS error with UKF is decreased with ratio about 50\%. Surely, adding the third sensor in a good alignment will give better results but in some problems like mobile robot adding a third sensor is meaningless. This means the solution is software by developing algorithms.

We used adaptive Kalman filter which is based on (IAE) and (AFKF) to solve measurement variance uncertainty with two sensors in linear states, and changed the flow chart of the algorithm by applying an adaptive nonlinear Kalman filter (ANKF), the results of ANKF1 and ANKF2 were better than EKF in view of RMS error, and the ANKF2 was the best. The following future work is to estimate the angular and angular rate for highly maneuvering targets in pitch and yaw with the nonlinear ANKF algorithms.

\section{REFERENCES}

[1] F. Sana, "Modeling and estimation for maneuvering target tracking with inertial systems using interacting multiple models," Universitat Politècnica de Catalunya, 2010.

[2] Y. Bar-Shalom, X. R. Li, and T. Kirubarajan, Estimation with applications to tracking and navigation: theory algorithms and software. John Wiley \& Sons, 2004.

[3] Z. Tang, C. Sun, and Z. Liu, "The tracking algorithm for maneuvering target based on adaptive kalman filter," Dianzi Keji- Electronic Science and Technology, vol. 26, no. 4, pp. 78-81, 2013.

[4] A. Toloei and S. Niazi, "State estimation for target tracking problems with nonlinear Kalman filter algorithms," International Journal of Computer Applications, vol. 98, no. 17, 2014.

[5] N. Al-Najdawi, S. Tedmori, E. A. Edirisinghe, and H. E. Bez, "An automated real-time people tracking system based on KLT features detection," Int. Arab J. Inf. Technol., vol. 9, no. 1, pp. 100-107, 2012.

[6] J. L. Anderson, "An ensemble adjustment Kalman filter for data assimilation," Monthly weather review, vol. 129, no. 12, pp. 2884-2903, 2001.

[7] S. Nardone, A. Lindgren, and K. Gong, "Fundamental properties and performance of conventional bearings-only target motion analysis," IEEE Transactions on automatic control, vol. 29, no. 9, pp. 775-787, 1984.

[8] J. Hartikainen, A. Solin, and S. Särkkä, "Optimal filtering with Kalman filters and smoothers," Department of Biomedica Engineering and Computational Sciences, Aalto University School of Science, 16th August, 2011.

[9] Y. Zhou, C. Zhang, Y. Zhang, and J. Zhang, "A new adaptive square-root unscented Kalman filter for nonlinear systems with additive noise," International Journal of Aerospace Engineering, vol. 2015, 2015.

[10] K. P. B. Chandra, D.-W. Gu, and I. Postlethwaite, "Cubature Kalman filter based localization and mapping," in World Congress, 2011, vol. 18, no. 1, pp. 2121-2125.

[11] I. Arasaratnam and S. Haykin, "Cubature Kalman filtering: A powerful new tool for aerospace applications," ed, 2009.

[12] P. H. Leong, S. Arulampalam, T. A. Lamahewa, and T. D. Abhayapala, "Gaussian-sum cubature Kalman filter with improved robustness for bearings-only tracking," IEEE Signal Processing Letters, vol. 21, no. 5, pp. 513-517, 2014.

[13] M. Oussalah and J. De Schutter, "Adaptive Kalman filter for noise identification," in Proceedings of the International Seminar on Modal Analysis, 2001, vol. 3, pp. 1225-1232: Citeseer.

[14] F. Deng, J. Chen, and C. Chen, "Adaptive unscented Kalman filter for parameter and state estimation of nonlinear high-speed objects," Journal of Systems Engineering and Electronics, vol. 24, no. 4, pp. 655-665, 2013.

[15] Siti Nurmaini, Bambang Tutuko, " Intelligent Robotics Navigation System: Problems, Methods, and Algorithm, " International Journal of Electrical and Computer Engineering (IJECE) Vol. 7, No.6, December 2017, pp. 3711 3726.

[16] Jinho Kim, Jangmyung Lee, " An Active Virtual Impedance Control Algorithm For Collision Free Navigation of a Mobile a Robot, "International Journal of Robotics and Automation (IJRA) Vol. 6, No. 2, June 2017, pp. 101 113.

[17] Bashar Alathari, Mohammed Falih Kadhim, Salam Al-Khammasi, Nabeel Salih Ali, " A framework implementation of surveillance tracking system based on pir motion sensors, " Indonesian Journal of Electrical Engineering and Computer Science (IJEECS) Vol. 13, No. 1, January 2019, pp. 235 242.

[18] Lasmadi, Adha Cahyadi, Samiadji Herdjunanto, Risanuri Hidayat, " Inertial Navigation for Quadrotor Using Kalman Filter with Drift Compensation," International Journal of Electrical and Computer Engineering (IJECE) Vol. 7, No. 5, October 2017, pp. 2596 2604.

[19] D.-J. Jwo and T.-P. Weng, "An adaptive sensor fusion method with applications in integrated navigation," The Journal of Navigation, vol. 61, no. 4, pp. 705-721, 2008. 